\title{
On Some Bending Problems of Prismatic Shell with the Thickness Vanishing at Infinity
}

\author{
Natalia Chinchaladze ${ }^{1}$ and Margarita Tutberidze ${ }^{2}$ \\ 1. I.Vekua Institute of Applied Mathematics \& Faculty of Exact and Natural Sciences of Iv. Javakhishvili Tbilisi State University, 2 \\ University St., 0186 Tbilisi, Georgia, email: chinchaladze@gmail.com \\ 2. I.Vekua Institute of Applied Mathematics \& Faculty of Exact and Natural Sciences of Iv. Javakhishvili Tbilisi State University, \\ email: tutberidze.rita@mail.ru
}

Received: December 05, 2016 / Accepted: January 03, 2017 / Published: March 25, 2017.

\begin{abstract}
The present work is devoted to the bending problems of prismatic shell with the thickness vanishing at infinity as an exponential function. The bending equation in the zero approximation of Vekua's hierarchical models is considered. The problem is reduced to the Dirichlet boundary value problem for elliptic type partial differential equations on half-plane. The solution of the problem under consideration is constructed in the integral form.
\end{abstract}

Key words: Cusped prismatic shell, Vekua's hierachical models, Elliptic type partial differential equations

\section{Introduction}

In 1955 I.Vekua raised the problem of investigation of elastic cusped prismatic shells, whose thickness on the prismatic shell entire boundary or on its part vanishes (see [17], [10]). In practice, such cusped prismatic shells, in particular, cusped plates, and cusped beams (i.e.,beams whose cross-sections area vanishes at least at one end of the beam) are often encountered in spatial structures with partly fixed edges, e.g., stadium ceilings, aircraft wings, submarine wings etc., in machine-tool design, in astronautics, turbines, and in many other applied fields of engineering. Investigation of elastic cusped prismatic shells, considered as 3D ones, may occupy 3D domains with, in general, non-Lipschitz boundaries. The problem mathematically leads to the question of setting and solving of boundary value problems for even order

Corresponding author: Natalia Chinchaladze, Ph.D. (Candidate of Sci.) in Mechanics of Deformable Bodies, Associated Prof., research field: Partial Differential Equations; Mathematical Modeling; Shell and Plate Theory, Cusped Plates and Shells. E-mail: chinchaladze@gmail.com. equations and systems of elliptic type with the order degeneration in the statical case and of initial boundary value problems for even order equations and systems of hyperbolic type with the order degeneration in the dynamical case. The cusped plates within the framework of the classical bending theory were first considered by Makhover and Mikhlin in [14], [15]. Problems for cusped plates have been investigated by Khvoles, Jaiani, Tsiskaridze, Khomasuridze, Devdariani, Uzunov, Naguleswaran, Kharibegashvili, Natroshvili, Wendland, and others (see, e.g. [10], [11], [13], [8], [9] and references therein).

At the same time Vekua introduced a new mathematical model for elastic prismatic shells which was based on expansions of the three-dimensional displacement vector fields and the strain and stress tensors in linear elasticity into orthogonal Fourier-Legendre series with respect to the variable plate thickness [18]. By taking only the first $N+1$ terms of the expansions, he introduced the so called $N$-th approximation. Each of these approximations for $N=0 ; 1 ; \ldots$ can be considered as an independent 
mathematical model of plates. In particular, the approximation for $N=0$ coincides with plane stress and plane deformation, $N=1$ corresponds to the classical Kirchhoff plate model [10]. Works of Babuska, Gordeziani, Guliaev, Khoma, Khvoles, Meunargia, Schwab, Vashakmadze, Zhgenti, Jaiani, Tsiskarishvili, M. and G. Avalishvili, Wendland, Natroshvili, Kharibegashvili, Chinchaladze, Gilbert, and others are devoted to further analysis of I.Vekua's models (rigorous estimation of the modeling error, numerical solutions, etc.) and their generalizations (see, e.g., [2], [3], [4], [5], [6], [7], [8], [9], [12], [16]).

In what follows, $\sigma_{i j}$ and $e_{i j}$ are the stress and strain tensor, respectively, $u_{i}$ are displacements, $\Phi_{i}$ are volume force components, $\rho$ is the density, $\lambda$ and $\mu$ are the Lamé constants, $\delta_{i j}$ is Kroneker delta. Moreover, repeated indices imply summation, the bar under one of the repeated indices means that we do not sum, indices after comma mean differentiation with respect to this value.

Let a $3 D$ elastic body occupy a bounded region $\bar{\Omega}$ with boundary $\partial \Omega$ :

$$
\begin{gathered}
\Omega:=\left\{\left(x_{1}, x_{2}, x_{3}\right) \in R^{3}: x:=\left(x_{1}, x_{2}\right) \in \omega,\right. \\
\left.\stackrel{(-)}{h}(x)<x_{3}<\stackrel{(+)}{h}(x)\right\},
\end{gathered}
$$

where $\bar{\omega}=\omega \cup \partial \omega$ is the so-called projection of the plate $\bar{\Omega}=\Omega \cup \partial \Omega$.

We assume that $\stackrel{(-)}{h}(x) \in C^{2}(\omega) \cap C(\bar{\omega})$ and the thickness is

$$
2 h(x):=\stackrel{(+)}{h}(x)-\stackrel{(-)}{h}(x)>0 \text { for } x \in \omega
$$

and

$$
2 h(x):=\stackrel{(+)}{h}(x)-\stackrel{(-)}{h}(x) \geq 0 \text { for } x \in \partial \omega,
$$

i.e., the thickness may vanish on some part of the boundary.
In the zero approximation of Vekua's hierarchical methods the governing system has the following form (see, e.g. [10], [18])

$$
\begin{aligned}
& \mu\left[\left(h v_{\alpha 0, \beta}\right)_{, \beta}+\left(h v_{\beta 0, \alpha}\right)_{, \beta}\right]+\lambda \delta_{\alpha \beta}\left(h v_{\gamma 0, \gamma}\right)_{, \beta} \\
& +\Phi_{\alpha 0}+\left(Q_{n \alpha}^{(+)}+\underset{n \alpha}{Q_{(-)}}\right) \\
& \times \sqrt{\left(h_{, 1}\right)^{2}+\left(h_{, 2}\right)^{2}+1}=0, \quad \alpha=1,2, \\
& \mu\left(h v_{30, \beta}\right)_{, \beta}+\Phi_{30}+\left(Q_{n 3} Q_{n 3}+\underset{(-)}{Q_{n}}\right) \\
& \times \sqrt{\left(h_{, 1}\right)^{2}+\left(h_{, 2}\right)^{2}+1}=0,
\end{aligned}
$$

where

$$
v_{i 0}:=\frac{u_{i 0}}{h}, i=1,2,3,
$$

$u_{i 0}$ and $\Phi_{i 0}$ are the zeroth moments of the $3 \mathrm{D}$ displacement vector and volume force components respectively, i.e.,

$$
\begin{aligned}
& \left(u_{10}, \Phi_{i 0}\right):=\int_{\substack{(-) \\
h(x)}}^{\substack{(+) \\
(x)}}\left(u_{i}, \Phi_{i}\right) d x_{3}, i, j=1,2,3,
\end{aligned}
$$

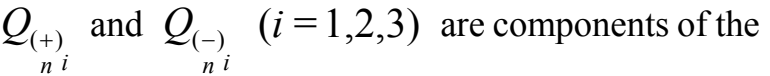
stress vectors acting on the upper and lower face surfaces with normals $\stackrel{(+)}{n}$ and $\stackrel{(-)}{n}$, respectively.

We consider the cusped prismatic shell with the half thickness as follows

$$
\begin{gathered}
h=h_{0} e^{-k x_{2}}, h_{0}=\text { const }>0, \kappa=\text { const } \geq 0, \\
-\infty<x_{1}<+\infty, 0 \leq x_{2}<+\infty
\end{gathered}
$$

The upper and lower surfaces of the plate under consideration are given in Fig.1. On Fig.2 and 3 the projection of the plate on the planes $0 x_{1} x_{3}$ and profile are given, respectively. 


\section{Cylindrical Bending}

Let us consider the case when all mechanical quantities depend on one space variable $x_{2}$. System (2) can be rewritten in the vector form as follows:

$$
v_{, 22}-\alpha v_{, 2}=f,
$$

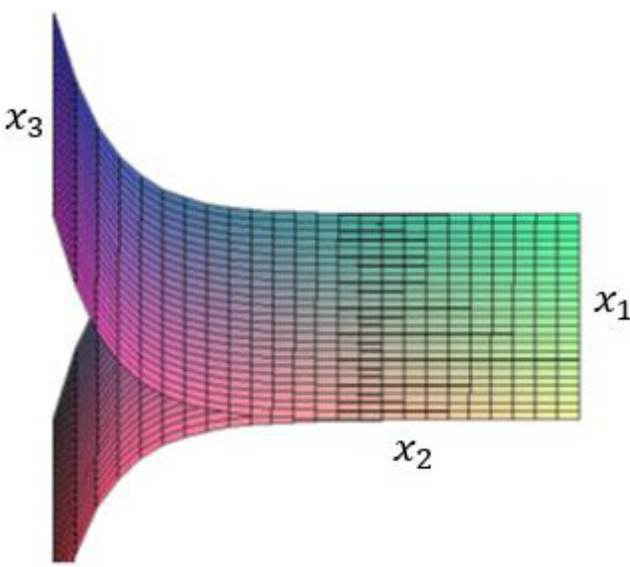

Fig. 1 The upper and lower surfaces of the plate.

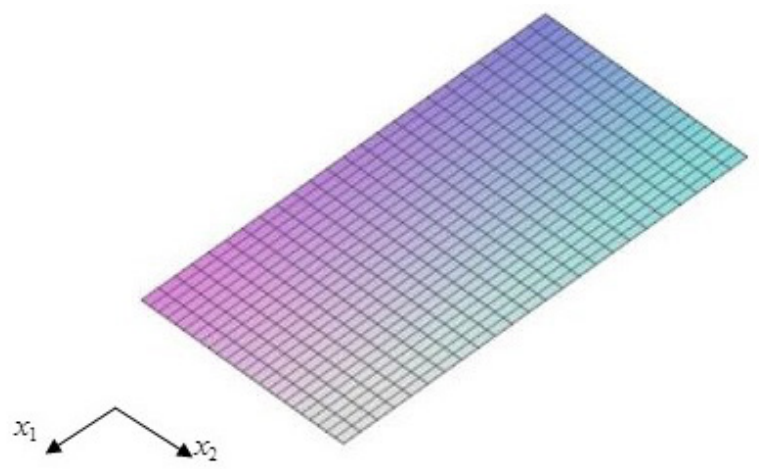

Fig. 2 The prjection of the plate on $O x_{1} x_{3}$.

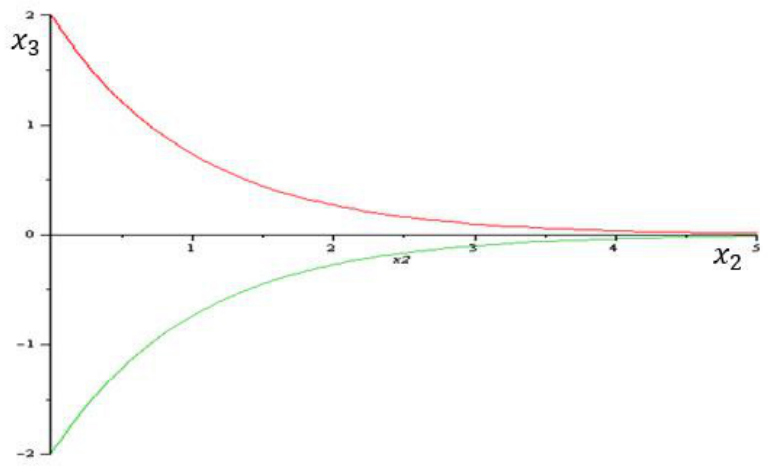

Fig. 3 The profile of the plate on $O x_{2} x_{3}$. where

$$
\begin{gathered}
v:=\left(v_{10}, v_{20}, v_{30}\right)^{T}, \\
f=\left(f_{10}, f_{20}, f_{30}\right)^{T}, \\
f_{10}:=-\frac{1}{\mu h_{0}} F_{10} e^{\alpha x_{2}}, \\
f_{20}=-\frac{1}{(\lambda+2 \mu) h_{0}} F_{20} e^{\alpha x_{2},}, \\
f_{30}=-\frac{1}{\mu h_{0}} F_{30} e^{\alpha x_{2}} .
\end{gathered}
$$

Problem 1. Find a solution of (4) under the following conditions

$$
\begin{gathered}
v(0)=v^{0}, v^{0}=\text { const, } \\
\left|v\left(x_{2}\right)\right|=O(1), x_{2} \rightarrow \infty, \\
v \in C^{2}(] 0 ;+\infty[) \cup C^{1}([0 ;+\infty[) .
\end{gathered}
$$

The solution of Problem 1 has a form

$$
\begin{gathered}
v\left(x_{2}\right)=-\frac{e^{\alpha x_{2}}}{\alpha} \int_{x_{2}}^{\infty} f d \xi \\
-\frac{1}{\alpha} \int_{0}^{x_{2}} f e^{\alpha \xi} d \xi+\frac{1}{\alpha} \int_{0}^{\infty} f d \xi+v^{0} .
\end{gathered}
$$

The sufficient conditions for fulfilment of (5) are

$$
\begin{gathered}
f\left(x_{2}\right)=\frac{B\left(x_{2}\right)}{\left(x_{2}+c\right)^{\beta}} e^{-\alpha x_{2}}, \\
\beta>1, c>0, B\left(x_{2}\right) \in C([0 ;+\infty)) .
\end{gathered}
$$

\section{Bending Problem}

In view of (16) equation (2) can be rewritten as follows

$$
\mu e^{-\kappa x_{2}}\left(v_{30,11}+v_{30,22}-\kappa v_{30,2}\right)+F_{30}=0,
$$

where 


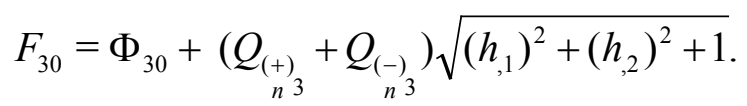

Let us consider the following problem:

Problem. Find a solution of equation (6) under the following boundary conditions

$$
v_{30}\left(x_{1}^{0}, x_{2}^{0}\right)=0, \quad x:=\left(x_{1}^{0}, x_{2}^{0}\right) \in \partial \omega,
$$

and condition at infinity

$$
\begin{gathered}
v_{30}=O\left(e^{k x_{2}}\right), \\
x_{2} \rightarrow+\infty,
\end{gathered}
$$

where

$$
\begin{gathered}
v_{30} \in C^{2}(\omega) \cap C(\bar{\omega}), \quad F_{30} \in C([0, \ell]), \\
\omega:=\left\{\left(x_{1}, x_{2}\right):-\infty<x_{1}<+\infty ; 0<x_{2}<+\infty\right\} .
\end{gathered}
$$

Let us rewrite equation (6) in the following form

$$
v_{30,11}+v_{30,22}-\kappa v_{30,2}=F,
$$

where

$$
F:=-\frac{1}{\mu} F_{30} e^{\kappa x_{2}}, \kappa>0 .
$$

After introducing new independent variables $z:=x_{1}+i x_{2}$ and $\zeta:=x_{1}-i x_{2}$, equation (9) and boundary conditions (7) can be rewritten in the complex form as follows

$$
\begin{gathered}
L(U(z, \zeta))=\frac{\partial^{2} U(z, \zeta)}{\partial z \partial \zeta}+A \frac{\partial U(z, \zeta)}{\partial z} \\
+B \frac{\partial U(z, \zeta)}{\partial \zeta}-F_{1}(z, \zeta)=0 \\
U\left(z, \zeta_{0}\right)=0, U\left(z_{0}, \zeta\right)=0 \\
z_{0}:=x_{1}^{0}+i x_{2}^{0}, \zeta_{0}:=x_{1}^{0}-i x_{2}^{0},
\end{gathered}
$$

where

$$
F_{1}(z, \zeta):=\frac{1}{4} F\left(\frac{z+\zeta}{2}, \frac{z-\zeta}{2 i}\right) .
$$

and

$$
\begin{gathered}
A:=-i \frac{\kappa}{4}, \quad B:=i \frac{\kappa}{4} \\
U(z, \zeta):=v_{30}\left(\frac{z+\zeta}{2}, \frac{z-\zeta}{2 i}\right)
\end{gathered}
$$

are analytic in some domain $\left(D^{*}, \bar{D}^{*}\right)$, where $z \in D^{*}, \zeta \in \bar{D}^{*}$.

The conjugate homogeneous equation of equation (10) has the form

$$
L^{*}(V(z, \zeta))=\frac{\partial^{2} V(z, \zeta)}{\partial z \partial \zeta}
$$

$$
-A \frac{\partial V(z, \zeta)}{\partial z}-B \frac{\partial V(z, \zeta)}{\partial \zeta}=0
$$

As usual, under the symbols $\frac{\partial}{\partial z}$ and $\frac{\partial}{\partial \zeta}$ we assume

$$
\frac{\partial}{\partial z}:=\frac{1}{2}\left(\frac{\partial}{\partial x_{1}}-i \frac{\partial}{\partial x_{2}}\right), \frac{\partial}{\partial \zeta}:=\frac{1}{2}\left(\frac{\partial}{\partial x_{1}}+i \frac{\partial}{\partial x_{2}}\right) .
$$

It can be proved that the solution of equation (12) has the following form

$$
V:=R(z, \zeta ; t, \tau)=e^{-i \frac{\kappa}{2}(\zeta-\tau)+i \frac{K}{2}(z-t)} .
$$

Using the last expression, the solution of equation (10) can be written as follows ([1], [19])

$$
U(z, \zeta)=\int_{z_{0}}^{z} \int_{\zeta_{0}}^{\zeta} e^{i \frac{\kappa}{2}(\zeta-\tau)-i \frac{\kappa}{2}(z-t)} F_{1}(t, \tau) d \tau d t
$$

where

$$
z_{0}:=x_{1}^{0}+i x_{2}^{0}, \quad z_{0}:=x_{1}^{0}-i x_{2}^{0}, \quad\left(x_{1}^{0}, x_{2}^{0}\right) \in \partial \omega .
$$

Let at first $F_{1}(t, \tau) \equiv 1$. From (13) we have

$$
U(z, \zeta)=\int_{z_{0}}^{z} e^{-i \frac{\kappa}{2}(z-t)} d t \int_{\zeta_{0}}^{\zeta} e^{i \frac{\kappa}{2}(\zeta-\tau)} d \tau
$$

$$
=\frac{4}{\kappa^{2}}\left[1-e^{-i \frac{\kappa}{2}\left(z-z_{0}\right)}\right]\left[1-e^{\frac{i \kappa}{2}\left(\zeta-\zeta_{0}\right)}\right] .
$$


Thus, by virtue of (14) we have the following estimate

$$
\left|v_{30}\left(x_{1}, x_{2}\right)\right| \leq \frac{2}{\kappa^{2}}\left[1+2 e^{\frac{\kappa}{2}\left(x_{2}-x_{2}^{0}\right)}+e^{\kappa\left(x_{2}-x_{2}^{0}\right)}\right]
$$

So, from (15) we get

$$
v_{30}(x)=O\left(e^{\kappa x_{2}}\right) \text {, when } x_{2} \rightarrow+\infty \text {. }
$$

Let $F_{1}(t, \tau)$ be an arbitrary continuous bounded function $\left(\left|F_{1}(t, \tau)\right| \leq M=\right.$ const $\left.<\infty\right)$, consequently

$$
\begin{gathered}
|U(z, \zeta)|=\left|\int_{z_{0}}^{z} \int_{\zeta_{0}}^{\zeta} e^{i \frac{\kappa}{2}(\zeta-\tau)-i \frac{\kappa}{2}(z-t)} \cdot F_{1}(t, \tau) d \tau d t\right| \\
\leq \int_{z_{0}}^{z} \int_{\zeta_{0}}^{\zeta}\left|e^{i \frac{\kappa}{2}(\zeta-\tau)-i \frac{\kappa}{2}(z-t)} \| F_{1}(t, \tau)\right| d \tau d t \\
\leq M \cdot \int_{z_{0}}^{z} \int_{\zeta_{0}} e^{i \frac{\kappa}{2}(\zeta-\tau)-i \frac{\kappa}{2}(z-t)} d \tau d t .
\end{gathered}
$$

Therefore, (13) is a solution of the setting problem.

\section{Conclusions}

Bending problem of the cusped symmetric prismatic shells (plates) with the half thickness as follows

$$
\begin{gathered}
h=h_{0} e^{-\kappa x_{2}}, \\
h_{0}=\text { const }>0, \kappa=\text { const } \geq 0, \\
-\infty<x_{1}<+\infty, 0 \leq x_{2}<+\infty .
\end{gathered}
$$

is considered. The problem mathematically is reduced to the Dirichlet boundary value problem for elliptic type partial differential equations on half-plane. Using complex representations the solution of the problem under consideration is constructed in the integral form.

\section{References}

[1] A.V. Bitsadze, Some Classes of Partial Differential Equations. Advanced Studies in Contemporary Mathematics 1988.
[2] N. Chinchaladze, Cylindrical bending of the prismatic shell with two sharp edges in the case of a strip. Rep. Enlarged Sess. Semin. I. Vekua Institute of Appl. Math. 10, No. 1 (1995) 21-23.

[3] N. Chinchaladze, Harmonic vibration of a cusped plates in the $N$-th approximation of Vekua's hierarchical models. Archives of Mechanics 65, No. 5 (2013) 345-366.

[4] N. Chinchaladze, On one problem of a cusped elastic prismatic shells in case of the third model of Vekua's hierarchical model. Journal of Mathematics and Statistics, Hacettepe University, airly access, Doi: 10.15672/HJMS.20164516346.

[5] N. Chinchaladze, R.P. Gilbert, Cylindrical vibration of an elastic cusped plate under action of an incompressible fluid in case of $\mathrm{N}=0$ approximation of I. Vekua's hierarchical models. Complex Variables 50, 7-11 (2005) 479-496.

[6] N. Chinchaladze, R.P. Gilbert, Harmonic vibration of prismatic shells in zero approximation of Vekua's hierarchical models. Applicable Analysis: An International Journal 90, No. 11 (2013) 2275-2287.

[7] N. Chinchaladze, G. Jaiani, R. Gilbert, S, Kharibegashvili, D. Natroshvili, Existence and uniqueness theorems for cusped prismatic shells in the N-th hierarchical model. Mathematical Methods in Applied Sciences 31, No. 11 (2008) 1345-1367.

[8] G. Devdariani, The first boundary value problem for a degenerate elliptic system. Bulletin TICMI 5 (2001) 23-24.

[9] G.V. Jaiani, S.S. Kharibegashvili, D.G. Natroshvili, W.L. Wendland, Twodimensional Hierarchical Models for Prismatic Shells with Thickness Vanishing at the Boundary. Journal of Elasticity 77, No. 2 (2004) 95-122.

[10] G. Jaiani, Cusped shell-like structures. SpringerBriefs in Applied Science and Technology, Springer, Heidelberg, 2011.

[11] G. Jaiani, Theory of cusped Euler-Bernoulli beams and Kirchoff-Love plates. Lect. Notes of TICMI 3, 2002.

[12] G.V. Jaiani, B.W. Schulze, Some degenerate elliptic systems and applications to cusped plates. Mathematische Nachrichten 280, No. 4 (2007) 388-407.

[13] A.R. Khvoles, The general representation for solutions of equilibrium equations of prismatic shell with variable thickness. Seminar of the Institute of Applied Mathematics of Tbilisi State University, Annot. of Reports 5 (1971) 19-21 [in Russian].

[14] E. Makhover, Bending of a plate of variable thickness with a cusped edge. Scientific Notes of Leningrad State Ped. Institute 17, 2 (1957) 28-39 [in Russian].

[15] S.G. Mikhlin, Variational methods in mathematical physics. Nauka, Moscow 1970 [in Russian].

[16] D. Natroshvili, S. Kharibegashvili, Investigation of 
hyperbolic systems with order degeneration arising in I. Vekua's hierarchical models, Applicable Analysis: An International Journal 92, No. 12 (2013) 2508-2525

[17] I.N. Vekua, On a method of computing prismatic shells, Akad. Nauk Gruzin. SSR. Trudy Tbiliss. Mat. Inst. Razmadze 21 (1955) 191-259 [in Russian].
[18] I.N. Vekua, Shell Theory: General Methods of Construction. Monographs, Advanced Texts and Surveys in Pure and Applied Mathematics, 25, Pitman (Advanced Publishing Program), Boston, MA. 1985.

[19] I.N. Vekua, New Methods for Solving Elliptic Equations. North-Holland Publ. Co., Amsterdam, 1967. 